(2) SMALL RENAL MASSES

\section{Jury still out on robotic partial nephrectomy}

At the recent congress of the European Association of Urology in Milan, Italy, much time was given to demonstrations and discussions of robot-assisted surgery for indications such as kidney and prostate cancer. Arguably, the use of robotic surgery is the most talked about advancement in modern urological practice, with many vocal proponents vehemently insisting that the technique is superior to laparoscopic techniques because it reduces tremor and can provide a $3 \mathrm{D}$ view of the surgical field.

By contrast, others take the measured view that robot-assisted surgeries are not necessarily better but are much more expensive, with instrument maintenance quoted being as high as US\$100,000 per year.

\section{4 ....although robotic surgery is not superior to laparoscopy, it can safely be used... 77}

In lieu of randomized controlled trials that directly compare the techniqueswhich would be difficult to realize for numerous reasons-Kamran Ahmed and colleagues at Guy's Hospital in London, UK, conducted a systematic review of the literature to determine whether any differences between robotic and laparoscopic partial nephrectomy could be determined.

"Over the past decade, we have seen quite a rapid evolution in terms of identifying and managing small renal masses," Ahmed explains. Simultaneously, the use of robotic surgery has expanded dramatically, with most of the evidence for its rapid uptake coming from comparative studies from single centres.

Data from six reports were used for the meta-analysis, encompassing 92 patients who underwent robot-assisted surgery and 140 who had the laparoscopic procedure, with a total of 256 operations in the cohort. The researchers examined a range of variables, including selection criteria, operating time, blood loss, warm ischaemia time and length of hospital stay. No significant differences in blood loss or warm ischaemia time could be determined between patients who underwent the laparoscopic or robotic procedures. Furthermore, the lengths of stay and postoperative complication rates were equivalent.

"Given these findings, we reanalysed the data using Bland-Altman analysis to assess whether robotics can be used instead of laparoscopy," continues Ahmed. "We established that, although robotic surgery

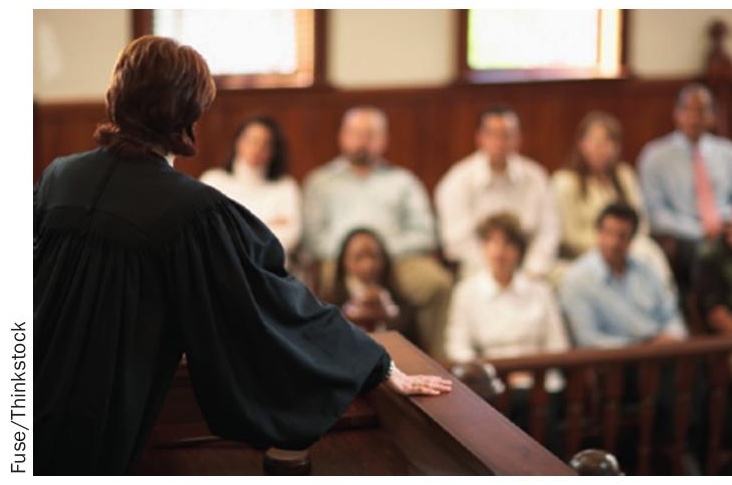

is not superior to laparoscopy, it can safely be used instead of laparoscopic surgery in selected patients."

Importantly, this study did not account for the complexity of the operations; for example, the tumour location might influence the patient outcomes. However, the results do suggest that more information is needed before robotic surgery is adopted as the standard of care for these patients. "As new techniques are being developed and more trainees become robot-competent, the outcomes will likely improve and we will see a shift towards management of renal masses with robotic techniques," predicts Ahmed.

Mina Razzak

Original article Froghi, S. et al. Evaluation of robotic and laparascopic partial nephrectomy for small renal tumours (T1a). BJU Int. doi:10.1111/bju.12053 